\title{
Bacterial nanotechnology
}

\author{
The 2018 Kavli Prize recognizes scientists working in the field of basic biology and reminds us of the importance of \\ fundamental research for uncovering the technologies of the future.
}

T he Kavli Prize was established in 2008 by philanthropist and physicist Fred Kavli, and is awarded every two years by the Kavli Foundation, in association with the Norwegian Academy of Science and Letters, to researchers working in the areas of nanoscience, neuroscience and astrophysics. The biographies and careers of the previous awardees of the Kavli Prize in Nanoscience, physicists and chemists who have devoted their lives to manipulate matter and light at the nanoscale, tell a story that is quintessentially nanotechnological.

With its roots in bacterial biology, this year's Nanoscience Kavli award appears to depart slightly from the previous ones. This year's prize invokes a tale of microscopic invasion and resistance, of a battle taking place at the nanoscale between bacteria and virus, and of human curiosity and inventiveness to understand first and to harness then the tools used in this battle. The 2018 Kavli Prize winners are Jennifer Doudna, Emmanuelle Charpentier and Virginijus Šikšnys, a structural biologist, a microbiologist and a biochemist, for their work on CRISPR-Cas9.

As for every scientific discovery, the one of the CRISPR-Cas9 bacterial system is the result of the collective feat of numerous scientists that spanned almost 30 years of research. These efforts showed that CRISPR-Cas9 represents a general immune system used by bacteria to fight off a viral attack. Bacteria integrate pieces of viral DNA in their genomes after they are invaded, building a memory of the event that triggers the CRISPR-Cas9 machinery in successive invasions. For a long time, however, the molecular mechanism of how the system worked remained obscure. It was only in 2012 , in two seminal independent papers published a short time apart ${ }^{1,2}$, that the winners of this year's Kavli prize described how Cas9 nuclease, guided by two RNA molecules that could be artificially fused together, identifies specific DNA sequences from invading species and cleaves them. Subsequent work from other groups showed that the CRISPR-Cas9 system could be used to cut a DNA sequence of choice in eukaryotic cells as well. At this point the scientific community fully realized that at its disposal was a genome editing technology that could be exploited in a variety of applications.
The beauty, and power, of the technique resides in its relatively straightforward programmability and versatility, which makes it more accessible but also raises questions about lack of regulations on its use. While other gene editing techniques require laborious protein engineering efforts to adapt the system to different gene sequences, in CRISPR-Cas9 the information regarding its specificity are encoded in the guide RNA, which can be easily programmed to recognize any stretch of DNA. These characteristics have converted CRISPR-Cas9 into the molecular biology workhorse in labs worldwide, as, for example, it allows the investigation of gene function or the creation of specific animal models at a fraction of the time required by old-school protocols, speeding up research at unimaginable rates. A sustained growth in the number of scientific publications in the field has occurred since 2012, and new biotech and 'gene programming' companies delivering CRISPR kits or CRISPR-modified cell lines on demand have mushroomed. The promises of the new system are great. In medicine, for example, it might allow correction of faulty genes in otherwise incurable genetic diseases, and the first ex vivo genome editing clinical trials have already started in China for cancer treatment and will start in Europe and the United States for $\beta$-thalassemia and sickle cell anaemia. The technology might revolutionize other fields as well. In agriculture, CRISPR-Cas9 might deliver crops more resistant to adverse conditions, and in energy it might lead to genetically engineered organisms for increased production of biofuels. In the field of infectious diseases, the technology might facilitate gene drive, a technique that aims at controlling the number of pathogen-carrying organisms by spreading genome alteration in a population. For example, scientists seek to apply this technology to slash the population of malaria-transmitting mosquitoes, but there are worries about the effect that this might have on the ecosystem.

The application of CRISPR-Cas9 as a human genome editing tool poses serious social and ethical concerns among the scientific community, as its intentional or unintentional misuse and the advent of possible private interests could lead to disruptive and dire consequences. Since the methodology can be used to modify human embryos, strict regulations should be put in place to draw lines that define what can be done, and what should never be done with the technology. Indeed, a few years ago several scientists signed a moratorium to promote continuous discussion on the ethics involved in gene editing and to demand that certain CRISPR-Cas lines of investigation be paused until the system and its pitfalls are better elucidated and the regulatory apparatus catches up with research with appropriate guidelines and safety measures.

As the ethical debate continues and the number of patents exploiting this technology increases, some scientists are going back to the bench to figure out more about the basics of the system. In particular, researchers want to know how the system has evolved, as well as understand its offtarget effects and how to limit them. Some researchers have upgraded it to a yet more precise cutting device, engineering Cas 9 proteins that can edit a gene at the level of a single base ${ }^{3}$ (the so called base editors), and others have tweaked it for molecular biology applications that go beyond gene editing ${ }^{4}$. Moreover, different CRISPR-Cas systems have been identified, with distinct substrate preferences and functions. Recently, three different groups have demonstrated the application of CRISPR-Cas12 and CRISPRCas13 systems for detection of viral DNA at extremely high sensitivities, a property that could lead to the engineering of low-cost portable devices for detection of infectious diseases in resource-limited settings ${ }^{5-7}$.

The example of CRISPR-Cas shows that investing in basic science to understand the inner workings of the natural world is key to developing new solutions to human struggles. Nature is a trove of potential technologies - it's up to the persistent and creative mind of man to find them, and to harness them responsibly.

Published online: 6 June 2018 https://doi.org/10.1038/s41565-018-0177-0

\footnotetext{
References

1. Jinek, M. et al. Science 337, 816-821 (2012).

2. Gasiunas, G., Barrangou, R., Horvath, P. \& Siksnys, V. Proc. Natl Acad. Sci. USA 109, E2579-E2586 (2012).

3. Gaudelli, N. M. et al. Nature 551, 464-471 (2017).

4. Adli, M. Nat. Commun. 9, 1911 (2018).

5. Chen, J. et al. Science 360, 436-439 (2018).

6. Gootenberg, J. S. Science 360, 439-444 (2018).

7. Myhrvold, C. et al. Science 360, 444-448 (2018).
} 